\title{
Oferta de Exportação de Açúcar do Brasil
}

Lucilio Rogerio Aparecido Alves ${ }^{1}$

Mirian Rumenos Piedade Bacchi²

Resumo - 0 objetivo deste trabalho é estimar uma função de oferta de exportação brasileira de açúcar utilizando a metodologia de Auto-Regressão Vetorial com identificação pelo processo de Bernanke. Os dados utilizados são do período de outubro de 1995 a dezembro de 2002. A equação especificada para avaliar os impactos de variações nas condicionantes das exportações é fundamentada em um modelo teórico que tem como pressuposto que elas se constituem, em grande parte, no excedente do mercado interno. As propriedades de integração e co-integração das séries utilizadas no modelo foram consideradas na análise. Os resultados mostram que o aumento do preço de exportação e a desvalorização cambial causam significativo aumento das exportações brasileiras. Por outro lado, um aumento da renda interna e do preço doméstico tem reflexos negativos sobre o quantum exportado. Os efeitos mais expressivos de uma mesma variação percentual nas condicionantes das exportações sobre o quantum exportado de açúcar, ocorrem no caso da variável renda interna.

Palavras-chave: comércio internacional, mercado de açúcar, Brasil.

\footnotetext{
${ }^{1}$ Doutorando do Programa de Pós-graduação em Economia Aplicada da Escola Superior de Agricultura "Luiz de Queiroz" - ESALQ/USP. Pesquisador do Centro de Estudos Avançados em Economia Aplicada - CEPEA/ESALQ/USP. Av. Pádua Dias, 11. CEP 13418-900 - Piracicaba - São Paulo. E-mail: lualves@ esalq.usp.br

2 Professora do Departamento de Economia, Administração e Sociologia - ESALQ/USP. Av. Pádua Dias, 11. CEP 13418-900 - Piracicaba - São Paulo. E-mail: mrpbacch@ esalq.usp.br
} 
Oferta de Exportação de Açúcar do Brasil

\section{1 - Introdução}

O objetivo deste trabalho é estimar uma função de oferta de exportação brasileira de açúcar utilizando a metodologia de Auto-Regressão Vetorial com identificação pelo processo de Bernanke. Os dados compreendem o período de outubro de 1995 a dezembro de 2002.

A balança comercial desempenha importante papel no processo de ajustamento das contas externas da economia nacional. Justifica-se a realização de análises que propiciem um maior conhecimento dos mecanismos responsáveis pela performance do segmento exportador da economia nacional, entre os quais encontram-se os de produtos agrícolas e agroindustriais.

A importância da agroindústria canavieira no contexto econômico nacional e dos produtos desse setor na balança comercial brasileira, entre os quais o açúcar é o que tem maior representatividade, motiva a condução de trabalhos que tratem do desempenho desse segmento exportador de forma particularizada. Estudos sobre as variáveis condicionantes das exportações brasileiras podem contribuir na definição de estratégias direcionadas à dinamização desses mercados. A estimação de equação de oferta de exportação de açúcar torna possível verificar o efeito de políticas de incentivo, possibilitando a elaboração de análises prospectivas sobre o comportamento do comércio externo, podendo também auxiliar os agentes ligados ao setor na tomada de decisões sobre produção e comercialização.

A relevância de estudar o funcionamento da agroindústria canavieira brasileira reside em sua grande importância tanto no contexto doméstico como internacional. No mercado doméstico, esta agroindústria diferencia-se dos demais países por produzir, em escala industrial, tanto açúcar como álcool. A cana-de-açúcar ocupa posição de destaque entre as principais culturas quanto à área plantada e também quanto ao volume e valor da produção.

No contexto internacional, a representatividade do produto brasileiro aumentou de forma expressiva nos últimos anos. Atualmente 0 Brasil é o maior produtor de açúcar do mundo, ultrapassando a produção da Índia e da União Européia e, no ano-safra 1995/ 96, tornou-se o maior exportador mundial desse produto, posição que mantém até os dias atuais. 
Além desta breve introdução, este trabalho consta de mais quatro outras partes. Na segunda, faz-se uma caracterização do setor açucareiro nacional. Na terceira, descreve-se o modelo econômico e, na quarta parte, os procedimentos metodológicos. Os dados utilizados e os resultados e discussão são apresentados na quinta parte do presente trabalho.

\section{2 - Caracterização dos Setores Exportador e Produtor de Açúcar Brasileiro}

É importante ressaltar dois momentos vividos pela agroindústria canavieira na década de 90 . 0 primeiro relacionado à extinção do Instituto do Açúcar e do Álcool (IAA), em março de 1990, que alterou a dinâmica do mercado de açúcar. Os produtores dessa commodity passaram a atuar, desde então, em um ambiente competitivo e foram forçados a empregar mecanismos de mercado nunca antes utilizados, quando o preço era fixado de forma a assegurar rentabilidade para a atividade. ${ }^{3}$ As dificuldades relacionadas a crédito, comuns em setores não regulados, estimularam os produtores a utilizar o mercado externo como forma de financiar a produção e aumentar a liquidez, sobretudo através de Adiantamentos sobre os Contratos de Câmbio (ACC) (Moraes, 2000). 0 período pós-desregulamentação coincide com aquele no qual tanto a produção como as exportações brasileiras cresceram, especialmente na Região Centro-Sul, a taxas superiores às praticadas anteriormente.

Um segundo momento está relacionado à mudança da política cambial brasileira, ocorrida em janeiro de 1999, quando foi extinto o chamado "regime de bandas cambiais", que determinava os limites de flutuação do preço da moeda estrangeira, adotando-se o regime de câmbio flexível. A liberalização do câmbio foi acompanhada por uma progressiva desvalorização da moeda brasileira, o que elevou a competitividade dos produtos nacionais exportáveis, favorecendo, entre outros, o setor exportador de açúcar.

\footnotetext{
${ }^{3}$ Destaca-se que não obstante a liberalização havida nos últimos anos no mercado interno de açúcar e álcool, o comércio internacional de açúcar ainda enfrenta barreiras estabelecidas por outros países. Este é o caso das importações de açúcar dos Estados Unidos e das exportações pela União Européia. No caso do álcool, as transações internacionais ainda são pouco representativas.
} 
Os principais fatores que impulsionaram o crescimento das exportações brasileiras na década de 1990 têm sido apontados como: a liberalização das exportações em julho de 1994, que encerrou o regime de quotas tarifárias, quando se taxava em $40 \%$ os volumes exportados superiores aos estabelecidos como quota; o aumento da demanda mundial, que se refletiu em condições favoráveis no mercado internacional; e a extinção de acordos especiais de comércio entre governos, que propiciou a entrada do Brasil em mercados até então fechados.

Segundo Veiga Filho (2000), pode-se creditar o crescimento das exportações brasileiras de açúcar a motivos de origem interna e externa. De origem externa, cita o fim do acordo bilateral entre a União das Repúblicas Socialistas Soviéticas e Cuba (URSS-Cuba), que possibilitou ao Brasil ocupar, em parte, o lugar de Cuba nesse mercado. No âmbito interno cita as crescentes exportações oriundas do Estado de São Paulo, respaldadas pelos baixos custos de produção em relação ao de outras regiões do Brasil e do mundo. A produção de cana-de-açúcar da Região Norte-Nordeste caracteriza-se por menor produção e maiores custos, comparativamente à Região Centro-Sul, que é conhecida por sua alta produtividade e excelente desenvolvimento ambiental, com considerável potencial de expansão.

A redução da demanda de álcool hidratado na década de 1990 pode ser considerada outro fator que propiciou o aumento das exportações brasileiras de açúcar. Este fator conduz ao deslocamento da oferta, favorecendo a produção de açúcar em detrimento ao álcool.

De acordo com dados da safra 2001/02, o Brasil foi o principal produtor mundial seguido pela Índia, União Européia, China, EUA, Tailândia, México, Austrália e Cuba (United States Department of Agriculture - USDA, 2002). Nessa safra, a produção e exportação brasileiras representaram $15,2 \%$ e $28,7 \%$, respectivamente, do total mundial. Da safra 1992/ 93 à 2001/02, a produção mundial cresceu à taxa geométrica de $2,4 \%$ a.a. e a brasileira à taxa de 8,7\% a.a.. Quanto à exportação, o comércio mundial teve uma ampliação à taxa de 4,0\% a.a., sendo que as exportações brasileiras aumentaram 17,7\% a.a.. No período que compreende os anos-safra 1992/93 a $2001 / 02$, as exportações brasileiras representaram em média $42,2 \%$ do total produzido no país (dados básicos de USDA, 2002). Do total exportado, a maior parte refere-se ao açúcar na forma bruta, sendo o restante da categoria de açúcar branco, que inclui o cristal e o refinado. A participação da 
receita de exportação de açúcar na receita total de exportações brasileiras apresentou uma média de 3,9\% nos últimos anos (Associação de Produtores de Álcool e Açúcar do Estado do Paraná - Alcopar, 2003).

As exportações brasileiras de açúcar podem também ser avaliadas de forma desagregada, em termos de açúcares (bruto e branco) e em termos de regiões exportadoras (Centro-Sul e Norte-Nordeste). Nas Figuras 1 e 2 observa-se a tendência crescente das vendas de açúcar ao mercado externo. No período compreendido entre janeiro de 1996 e dezembro de 2002, o volume das exportações de açúcar bruto representou aproximadamente $61,25 \%$ do total exportado (Figura 1). A quantidade exportada pela Região Centro-Sul apresenta destaque, com aproximadamente $75 \%$ do total exportado, sendo que alguns meses suas exportações representaram $100 \%$ das exportações brasileiras (Figura 2).

As exportações das Regiões Centro-Sul e Norte-Nordeste diferem em relação aos seus mercados de destino. O número de países que importaram açúcar da Região Norte-Nordeste manteve-se praticamente inalterado entre 1996 e 2002. As exportações dessa região são destinadas, em grande parte, aos Estados Unidos da América (EUA), uma vez que as quotas preferenciais concedidas por este país ao Brasil são apropriadas pelas usinas nordestinas. O Centro-Sul, por sua vez, apresentou uma expansão no número de mercados importadores entre 1996 e 2002.

Figura 1 - Exportações brasileiras dos açúcares bruto e branco, janeiro de 1996 a dezembro de 2002 (em toneladas).

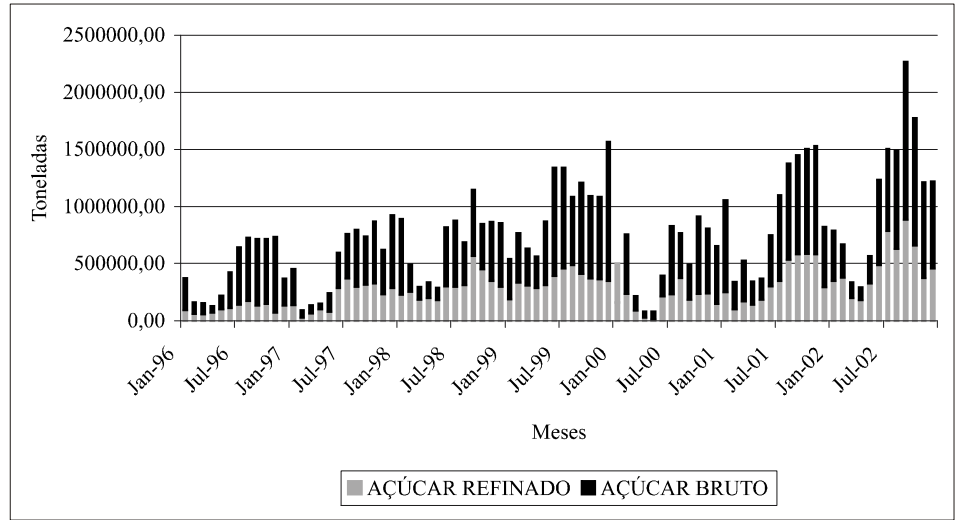

FONTE: MDIC/SECEX (2003). 
Figura 2 - Exportações totais de açúcar das Regiões Centro-Sul e Norte-Nordeste, janeiro de 1996 a dezembro de 2002 (em toneladas).

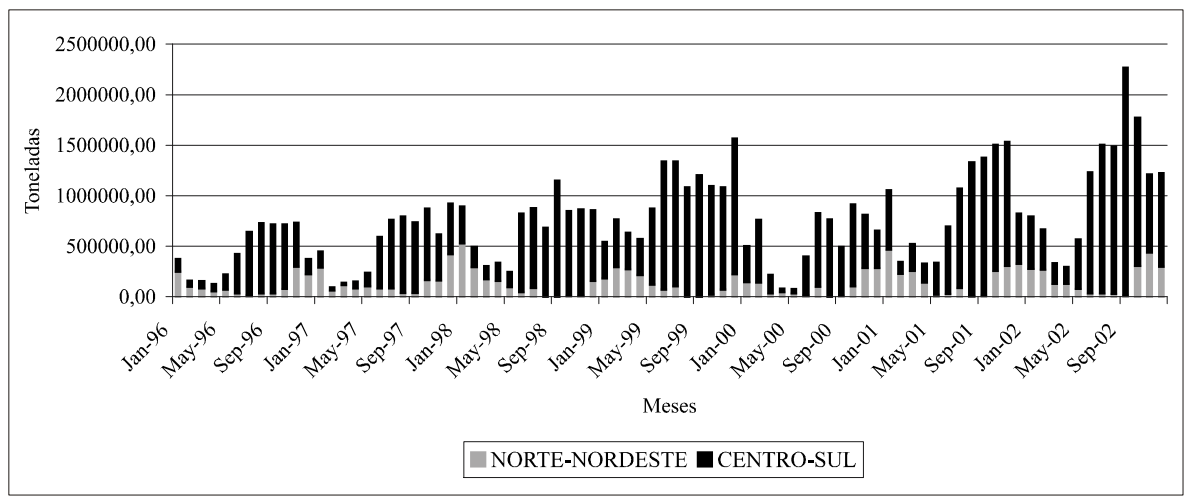

FONTE: MDIC/SECEX (2003).

Contudo, o aumento das receitas de exportação de açúcar está relacionado à possibilidade do Brasil ampliar sua participação no cenário mundial através da conquista de novos mercados. Embora as exportações brasileiras atinjam grande número de países, os sete principais destinos (Rússia, Nigéria, Emirados Árabes, Egito, Marrocos, Canadá e Irã) representam aproximadamente $63 \%$ do total exportado (dados básicos de Alcopar, 2003).

Destaca-se que uma das mais importantes características da agroindústria canavieira brasileira consiste na flexibilidade de sua produção. As usinas de açúcar com destilaria anexa podem direcionar o caldo oriundo da moagem da cana-de-açúcar tanto para a fabricação de açúcar como para de álcool, dependendo da rentabilidade desses produtos. Segundo Marjotta-Maistro (2002), no processo de tomada de decisão os agentes formam expectativa considerando preços e quantidades demandadas de açúcar e álcool, tanto no mercado interno como no externo. Além disso, por causa do grande crescimento da cana-de-açúcar baseada no suporte de ambas as indústrias (açúcar e álcool) no Brasil, o país demonstra o potencial de expansão da exportação de açúcar mais rápido do que qualquer outro país exportador.

Quanto ao mercado interno, os principais estados produtores de cana-de-açúcar, considerando a média da produção da safra 2002/03, 
são: São Paulo (60,6\%), Paraná (7,5\%), Alagoas (7,0\%), Pernambuco $(4,6 \%)$ e Minas Gerais (4,5\%). No geral, a agroindústria canavieira do Estado de São Paulo aparece como a mais dinâmica do país.

Entre as safras 1988/ 89 e 2001/02, a produção de açúcar da Região Norte-Nordeste cresceu à taxa de $0,9 \%$ a.a., enquanto que na Região Centro-Sul o crescimento foi de $11,5 \%$ a.a., resultando em uma expansão nacional de $8,6 \%$ a.a. (dados básicos da UNICA, 2002). Considerando a média das safras 1999/ 00 e 2001/02, a Região Centro-Sul produziu próximo de $83 \%$ do total nacional. 0 estado que mais se destaca nessa produção é São Paulo, que representou, na safra 2002/03, 60\% das produções da Região Centro-Sul e nacional.

Quanto às elasticidades da demanda de açúcar no mercado interno, no trabalho de Caruso (2000) foram estimadas as elasticidades-preço da ordem de -0,47 e -0,18, utilizando modelos ajustados por mínimos quadrados ordinários e mínimos quadrados generalizados, respectivamente. Sabe-se também que o açúcar é um produto que tem baixa elasticidade-renda da demanda. Hoffmann (2000), estimou, utilizando o ajustamento de poligonal com dados da POF de 1995/96, elasticidades-renda média da despesa com açúcares refinado e cristal da ordem de 0,13 e $-0,20$, respectivamente.

A literatura que trata do estudo do mercado do álcool, por sua vez, é ainda escassa em função da intervenção governamental verificada até passado recente. Poucos trabalhos sobre o processo de formação de preço no setor de combustíveis foram realizados no Brasil, podendo-se citar o de Marjotta-Maistro (2002). Essa autora estimou a elasticidade-renda da demanda de gasolina $\mathrm{C}$ em 0,21 e a elasticidade-preço em 0,6. Considerando que o álcool anidro é utilizado em proporções relativamente fixas na constituição da gasolina $C$, pode-se inferir que esses valores seriam uma boa proxy para as elasticidades de demanda de álcool anidro.

Observa-se, então, que tanto o açúcar como o álcool têm demandas inelásticas a preços e renda. Dessa forma, impactos nessas variáveis terão efeitos menos que proporcional na quantidade demandada dos produtos no mercado doméstico, podendo-se inferir que aumento na produção não levará, através da queda de preços, a aumentos significativos na demanda interna, especialmente no caso do açúcar, o que sugere a geração de excedentes que deverão ser direcionados ao mercado externo. 
O mercado exportador de açúcar brasileiro tem sido estudado sob vários enfoques e dentre os trabalhos existentes na literatura sobre esse mercado pode-se citar: Barros (1975), Carvalho \& Brandt (1987), Stalder \& Burnquist (1997), Reis \& Crespo (1998), Burnquist \& Miranda (1999), Caruso (2001) e Barros et al. (2002).

\section{3 - Modelo Econômico}

Muitos são os trabalhos existentes sobre o comércio internacional de commodities, alguns tratando de produtos no agregado, outros tratando da comercialização de produtos de forma individual. Entre aqueles que tratam de funções de exportação podem ser citados, entre outros: Goldstein \& Khan (1978), Zini (1988), Castro \& Cavalcanti (1997), Cavalcanti \& Ribeiro (1998), Onunkwo \& Epperson (1999), Senhadji \& Montenegro (1999), Carvalho \& Negri (2000), Miranda (2001) e Barros et al. (2002).

No presente estudo teve-se como objetivo estimar uma função de oferta de exportação de açúcar do Brasil, sendo a especificação dessa função feita fundamentando-se no modelo proposto por Barros et al. (2002). Na definição desse modelo, considera-se que o quantum exportado do produto depende dos excedentes do mercado doméstico.

As funções de oferta e demanda interna podem ser escritas na forma logarítmica, respectivamente, como:

$$
\begin{aligned}
& s=f(p d, w) \\
& d=g(p d, y)
\end{aligned}
$$

onde: pd é o logaritmo do preço doméstico; w representa deslocadores da oferta; e, y representa deslocadores da demanda, entre os quais podese citar a renda.

Considera-se que:

$$
q x=s-d
$$

onde qx é o quantum exportado.

Relacionando o preço das exportações e preço interno, tem-se uma margem de exportação que cobre o custo dessa operação. Admite-se 
que, na forma logarítmica, essa margem possa ser representada por:

$$
m=\alpha p d
$$

onde $\alpha$ é a elasticidade relacionando $\mathrm{m}$ a pd. 0 preço de exportação expresso em moeda nacional ( $p x$ ), na forma logarítmica, é igual ao preço que vigora no mercado interno mais a margem de exportação:

ou, ainda,

$$
\begin{aligned}
& p x=p d+m \text { ou } \\
& p d=p x-m
\end{aligned}
$$

$$
p d=p x-\alpha p d
$$

Tomando (1), (2) e (3), especifica-se a função de oferta de exportação como sendo afetada pelas mesmas variáveis que influenciam a oferta e demanda interna. Considerando também a relação expressa em (7), pode-se representar a função de oferta de exportação genericamente, em logaritmo, por:

$$
q x=h(p d, p x, w, y)
$$

Entretanto, sabendo-se que $p x=p e+t c$, onde pe é o logaritmo do preço das exportações em moeda estrangeira e tc é o logaritmo da taxa de câmbio, pode-se reescrever a equação (8) como

$$
q x=h(p e, t c, p d, w, y)
$$

Sendo o modelo expresso em logaritmos, os coeficientes estimados são as próprias elasticidades. Além do mais, a transformação dos dados em logaritmo ameniza problemas associados à variância não constante dos erros, quando eles existem.

No modelo apresentado, o quantum exportado depende dos preços de exportação expresso em moeda estrangeira, do preço doméstico, da taxa de câmbio real, da renda interna e de um deslocador da oferta (por exemplo, o álcool, não considerado neste trabalho por falta de séries 
temporais disponíveis). Esperam-se coeficientes com sinal negativo no caso do preço interno e da renda, e positivo para taxa de câmbio e preço das exportações. 0 preço interno tende a refletir o resultado do balanço entre a oferta e a demanda interna e, dessa forma, preços baixos refletem maior disponibilidade do produto e maior possibilidade de exportação. Uma mudança que provoque uma variação positiva no preço interno é um indicativo de que a demanda aumentou, relativamente à oferta, sinalizando uma queda das exportações; o inverso ocorreria no caso de queda no preço. Entretanto, espera-se que contemporaneamente haja influência apenas do preço de exportação sobre o preço doméstico e que os impactos dessas variáveis e das demais sobre o quantum a ser exportado ocorra apenas em períodos posteriores, conforme resultados de estudo preliminar realizado por Alves (2002).

Diferentemente do trabalho realizado por Barros et al. (2002), no qual a função de oferta de exportação de açúcar foi ajustada utilizando os procedimentos de Análise de Regressão clássicos, propôs-se aqui usar a metodologia de Auto-Regressão Vetorial para estimar aquela função, pois ela permite analisar os efeitos dinâmicos de alterações nas variáveis incluídas nos modelos. 0 modelo descrito foi usado como referência para a definição das variáveis relevantes da função de oferta de exportação. 0 procedimento de Auto-Regressão Vetorial permite que as variáveis incluídas no modelo sejam consideradas endógenas e, por serem especificadas nas defasagens, são tomadas como pré-determinadas.

\section{4 - Procedimentos Metodológicos}

A metodologia de Auto-Regressão Vetorial (VAR) foi proposta como alternativa aos modelos estruturais multi-equacionais. É uma abordagem bastante utilizada na análise de questões macroeconômicas e tem sido também utilizada em estudos relacionados à economia agrícola, podendo-se citar, nesse caso, Myers et al. (1990), Barros (1994), Burnquist et al. (1994), Aguiar (1994), Barros \& Bittencourt (1997) e Bacchi \& Burnquist (1999).

O uso da metodologia VAR permite a obtenção de elasticidades de impulso para k períodos à frente. Essas elasticidades de impulso possibilitam a avaliação do comportamento das variáveis em resposta a cho- 
ques (inovações) individuais em quaisquer dos componentes do sistema, podendo-se assim analisar, através de simulação, efeitos de eventos que tenham alguma probabilidade de ocorrer. A metodologia VAR possibilita a decomposição histórica da variância dos erros de previsão, $k$ períodos à frente, em percentagens a serem atribuídas a cada variável componente do sistema, analisando a importância de cada choque (em cada variável do modelo) ocorrido no passado na explicação dos desvios dos valores observados das variáveis em relação à sua previsão realizada no início do período considerado.

A metodologia VAR tem como uma limitação o fato de ter uma estrutura recursiva para as relações contemporâneas entre as variáveis. 0 modelo conhecido como VAR estrutural desenvolvido por Bernanke (1986) supera tal restrição e permite estabelecer relações contemporâneas tomando a teoria econômica como referência (Harvey, 1990 e Hamilton, 1994).

Um modelo VAR estrutural pode ser representado por:

$$
B_{0} \mathbf{y}_{t}=B_{1} \mathbf{y}_{t-1}+B_{2} y_{t-2}+\ldots+B_{p} y_{t-p}+e_{t}
$$

onde $\mathbf{y}_{\mathbf{t}}$ é um vetor com variáveis de interesse; $\mathbf{B}_{\mathbf{j}}$ são matrizes ( $n \times n$ ) para qualquer $\mathrm{j}$, com $\mathbf{B}_{\mathbf{0}}$ sendo a matriz de relações contemporâneas e $\mathbf{e}_{\mathbf{t}}$ é um vetor $\mathrm{n} \times 1$ de choques ortogonais. Além de se considerar que os componentes de $\mathbf{e}_{\mathbf{t}}$ são não correlacionados serialmente, adota-se a suposição de que eles não têm causa comum, tratando-os como mutuamente não correlacionados, de tal forma que $E\left(\mathbf{e}_{\mathbf{t}} \mathbf{e}_{\mathbf{t}}^{\prime}\right)=\mathbf{D}$. A equação (10) pode ser escrita como:

$$
\mathbf{B}(\mathrm{L}) \mathbf{y}_{t}=\mathbf{e}_{\mathbf{t}}
$$

onde $\mathbf{B}(\mathrm{L})$ é um polinômio em $\mathrm{L}\left(\mathbf{B}_{\mathbf{0}}+\mathbf{B}_{1} L+\mathbf{B}_{1} L^{2}+\ldots+\mathbf{B}_{\mathbf{p}} L^{P}\right)$ com $\mathrm{L}$ sendo o operador de defasagem tal que $L^{j} y_{t}=y_{t-j}$ para j inteiro.

Para fins de estimação, pré multiplica-se (11) por $\mathbf{B}_{0}^{-1}$ e obtém-se a forma reduzida:

$$
\mathbf{A}(\mathrm{L}) \mathbf{y}_{\mathbf{t}}=\mathbf{u}_{\mathbf{t}}
$$


onde $\mathbf{A}(\mathrm{L})=\mathbf{B}_{0}^{-1} \mathbf{B}(\mathrm{L}), \quad \mathbf{A}_{0}=\mathbf{I}_{\mathrm{n}}$ e $\mathbf{u}_{\mathbf{t}}=\mathbf{B}_{0}^{-1} \mathbf{e}_{\mathbf{t}}$. A equação (12) pode ser estimada por Mínimos Quadrados Ordinários. Com o uso do procedimento de Bernanke (1986) pode-se estimar, através da maximização da função de verossimilhança, os coeficientes de $\mathbf{B}_{\mathbf{0}}$ e $\mathbf{D}$.

Se o processo é estacionário (ver Lütkepohl, 1991), a equação (12) pode ser escrita na forma de média móvel.

$$
\mathbf{y}_{\mathbf{t}}=\mathbf{C}(\mathrm{L}) \mathbf{u}_{\mathrm{t}}
$$

onde $\mathbf{C}(L)$, que é estimado conhecendo-se $\mathbf{A}(L)$, é um polinômio de ordem infinita de matrizes $\mathbf{C}_{\mathbf{j}}$. Escrevendo a equação (13) em termos de $\mathbf{e}_{\mathbf{t}}$ tem-se

$$
\mathbf{y}_{\mathbf{t}}=\mathbf{C}(\mathrm{L}) \mathbf{B}_{0}^{-1} \mathbf{e}_{\mathbf{t}}
$$

Essa equação pode ser usada para analisar os efeitos dos choques e a decomposição da variância do erro de previsão. 0 modelo, conforme descrito, requer o uso de séries estacionárias ou séries que se tornam estacionárias após a diferenciação, objetivando evitar obter um relacionamento espúrio entre as variáveis. Para testar a estacionariedade das séries, utilizaram-se os testes de Dickey-Fuller. Se as séries são integradas de mesma ordem e co-integradas, um termo de correção de erro deve ser incluído no modelo, sem o que ocorre erro de especificação [mais detalhes em Engle \& Granger (1987) e Johansen \& Juselius (1990)].

Diversos estudos tratam de estabelecer procedimentos para verificar a ordem de integração de uma série temporal. Dentre os procedimentos existentes, os de Fuller (1976), complementados pelos de Dickey \& Fuller (1979 e 1981), tem sido bastante utilizados. Pressupondo que a série é gerada por um processo auto-regressivo de ordem $p[A R(p)], 0$ seguinte modelo pode ser utilizado para testar raiz unitária:

$$
\Delta x_{t}=\alpha+\beta T+\eta x_{t-1}+\sum_{i=1}^{p-1} \theta_{i} \Delta x_{t-1}+e_{t}
$$

sendo: $\eta=\sum_{i=1}^{p} \rho_{i}-1 ; \theta_{i}=-\sum_{j=i+1}^{p} \rho_{j} ;$ e $\mathrm{T}=$ tendência determinista do mo- 
delo. Os testes de AIC (AKAIKE Information Criterion) e SC (SCHWARZ Criterion) numa versão uni-equacional podem ser utilizados para a determinação do valor de $p$, de forma a se obter resíduos não correlacionados, ou seja, ruído branco (ver Lütkepohl, 1991). O teste Q de Ljung Box, por sua vez, dá uma indicação da existência ou não de autocorrelação serial, podendo ser utilizado como procedimento auxiliar na determinação do valor de $p$.

Os testes de Dickey \& Fuller consistem na utilização das estatísticas $\tau_{\beta \tau}$ e $\tau_{\alpha \mu}$ que avaliam a significância dos coeficientes da variável tendência (b) do modelo que inclui essa variável e da constante no modelo no qual a tendência é excluída. São utilizadas as estatísticas $\tau_{\tau}, \tau_{\mu}$ e $\tau$, as quais correspondem aos coeficientes da variável defasada $(\eta)$ do modelo com constante e tendência, apenas constante e sem constante e tendência, respectivamente.

O teste é repetido, quando necessário, fazendo-se diferenças sucessivas da série. 0 número de raízes unitárias (ordem de integração) é dado pelo número de vezes que a série deve ser diferenciada para se tornar estacionária.

Se as variáveis são integradas de mesma ordem, um próximo passo seria testar a existência de co-integração entre elas. 0 conceito de cointegração está relacionado com uma relação de equilíbrio no longo prazo entre as variáveis. A metodologia indicada para modelos com mais de duas variáveis explicativas, ou para aqueles nos quais existe endogeneidade dos regressores, é a proposta por J ohansen (1988).

0 procedimento de Johansen baseia-se na seguinte versão reparametrizada de um modelo de Vetor Auto-regressivo (VAR) de ordem $p$.

$$
? \mathbf{y}_{t}=G_{1} ? y_{t-1}+\ldots+G_{p-1} ? y_{t-p+1}+? y_{t-1}+\mu+\varphi d_{t}+e_{t}
$$

onde $\mathbf{y}_{\mathbf{t}}$ é um vetor com $\mathrm{k}$ variáveis, $\mathbf{e}_{\mathbf{t}} \sim \mathrm{N}(0, \Sigma)$ e $\mathrm{E}\left(\mathbf{e}_{\mathbf{t}} \mathbf{e}_{\mathrm{s}}^{\prime}\right)=0$ para qualquer $\mathrm{t}$ diferente de $\mathbf{s}$ e $\mathbf{d}_{\mathbf{t}}$ é um vetor de variáveis binárias para captar a variação estacional.

Considerando $r$ o posto da matriz ? , então ? tem $r$ autovalores diferentes de zero. Três situações podem ocorrer: se $r=k$ então $\mathbf{y}_{\mathbf{t}}$ é estacionário; se $r=0$ então $\Delta \mathbf{y}_{\mathbf{t}}$ é estacionário; finalmente, se $0<r<$ 
$\mathrm{n}$ existem matrizes $\mathbf{a}$ e $\mathbf{b}$ de dimensão $\mathrm{k} \times \mathrm{r}$ tais que $\boldsymbol{?}=\mathbf{a} \boldsymbol{\beta}^{\prime}$ e o vetor $\boldsymbol{B}^{\prime} \mathbf{y}_{\mathbf{t}}$ é estacionário, havendo $r$ vetores de co-integração (as $r$ colunas de b). Johansen $\&$ Juselius (1990) apresentaram dois testes, bem como seus valores críticos, para identificar o número de vetores de cointegração: teste do traço $\left(\boldsymbol{?}_{\text {trace }}\right)$ e do máximo autovalor $\left(?_{\max }\right)$. Os critérios AIC e SC, num contexto multi-equacional, são utilizados para a determinação do valor de $p$.

Para a realização dos testes de raiz unitária e de co-integração e para estimar o modelo VAR, utilizar-se-á o programa Regression Analysis of Time Series (RATS).

\section{5 - Resultados e Discussão}

Inicialmente, apresentam-se as fontes dos dados a serem utilizados no model o especificado para a análise da oferta de exportação brasileira de açúcar. Em seguida, descrevem-se os resultados dos testes efetuados.

Os dados de quantidade exportada (em sc/50kg) e de valor das exportações, utilizados no cálculo do preço das exportações (US\$/SC) $50 \mathrm{~kg}$ ), foram obtidos junto à Secretaria de Comércio Exterior (SECEX), do Ministério de Desenvolvimento Indústria e Comércio (MDIC). Os preços domésticos $(\mathrm{R} \$ \mathrm{sc} / 50 \mathrm{~kg})$ foram os divulgados pelo Centro de Estudos Avançados em Economia Aplicada, da Escola Superior de Agricultura "Luiz de Queiroz" (ESALQ/USP), deflacionados pelo Índice Geral de Preços - Disponibilidade Interna (IGP - DI), da Fundação Getúlio Vargas. A taxa de câmbio real utilizada é o índice calculado pelo Instituto de Pesquisa em Economia A plicada (IPEA), denominado Taxa de Câmbio Efetiva Real (IPA-OG - exportações). Para a renda foi usada como proxy o índice divulgado pelo Instituto Brasileiro de Geografia e Estatística - IBGE, da Pesquisa Industrial Mensal - Produção Física - Indústria Geral. Utilizou-se uma variável dummy para representar o período de safra e entressafra da cultura da cana-de-açúcar com a finalidade de melhorar o ajustamento do modelo, sabendo-se que as exportações são maiores no período de safra, que oficialmente compreende os meses de maio a dezembro. Os dados utilizados têm freqüência mensal e foram transformados em logaritmos para o ajustamento do modelo.

Na Tabela 1, apresentam-se os resultados dos testes de raiz unitária para 
as séries: quantidade exportada (qe), preço das exportações (pe), preço doméstico (pd), taxa de câmbio real (tc) e renda (ren). O procedimento proposto por Enders (1995) foi utilizado para identificar o modelo específico a ser considerado no teste, incluindo ou não termos deterministas. Os resultados encontrados apontam que no período analisado a série relativa à quantidade exportada é estacionária, enquanto as demais são integradas de ordem um [I(1)]. Portanto, é necessário que na especificação do modelo elas sejam consideradas nas diferenças de primeira ordem.

Nos resultados da análise de co-integração para as séries integradas de mesma ordem, os testes do máximo autovalor e do traço indicam que existe um vetor de co-integração (Tabela 2). Dessa forma, os resultados mostram que há relações de equilíbrio de longo prazo entre as variáveis. O modelo ajustado foi, portanto, um modelo VAR com Correção de Erro (VEC), considerando os aspectos tanto de curto quanto de longo prazos.

Tabela 1 - Resultados dos testes de raiz unitária para as variáveis que compõem o modelo especificado para a análise da oferta de exportação brasileira de açúcar

\begin{tabular}{cccccccc}
\hline Variá veis & Valor de & \multicolumn{3}{c}{ Modelo 1 * } & \multicolumn{1}{c}{ Modelo 2** $^{*}$} \\
\cline { 3 - 8 } & $\boldsymbol{p}$-1 & $\tau_{\tau}$ & $\tau_{\beta \tau}$ & $\tau_{\mu}$ & $\tau_{\alpha \mu}$ & $\tau$ & \multicolumn{1}{c}{$\tau$} \\
\hline$q e$ & 4 & $-4,698^{\#}$ & 2,103 & $-4,110$ & 4,123 & 0,340 & $-6,118^{\#}$ \\
$p e$ & 1 & $-1,913$ & $-1,564$ & $-1,095$ & 0,981 & $-1,075$ & $-10,056^{\#}$ \\
$p d$ & 2 & $-2,651$ & 0,701 & $-2,743$ & 2,739 & $-0,132$ & $-5,577^{\#}$ \\
$t c$ & 2 & $-2,635$ & 2,117 & $-1,607$ & 1,619 & 0,498 & $-6,869^{\#}$ \\
$r e n$ & 13 & $-2,141$ & 2,217 & $-0,557$ & 0,564 & 0,884 & $-2,223^{\# \#}$ \\
\hline
\end{tabular}

Fonte: Dados da pesquisa

\# Significativo ao nível de significância de 1\%. "\#ignificativo ao nível de 5\% de significância [valores críticos em Fuller (1976) e Dickey-Fuller (1981)].

* Modelo $1 \Rightarrow \Delta x_{t}=\alpha+\beta t+\gamma x_{t-1}+\sum_{i=1}^{p-1} \lambda_{i} \Delta x_{t-i}+\varepsilon_{t}$, nas versões com constante e tendência, sem tendência e sem tendência e constante.

** Modelo $2 \Rightarrow \Delta \Delta x_{t}=\gamma \Delta x_{t-1}+\sum_{i=1}^{p-2} \lambda_{i} \Delta \Delta x_{t-i}+\varepsilon_{t}$, definido após constatado a não existência de termos deterministas.

Obs.: não houve presença de autocorrelação serial, conforme o teste Q de Ljung Box. 
Tabela 2 - Resultados dos testes de co-integração de J ohansen

\begin{tabular}{cccc}
\hline Hipótese Nula & Hipótese Alternativa & $\boldsymbol{\lambda}_{\max }$ & $\boldsymbol{\lambda}_{\text {trace }}$ \\
\hline $\mathrm{r} \leq 3$ & $\mathrm{r}=4$ & 3,342 & 3,342 \\
$\mathrm{r} \leq 2$ & $\mathrm{r}=3$ & 8,485 & 11,826 \\
$\mathrm{r} \leq 1$ & $\mathrm{r}=2$ & 14,908 & 26,734 \\
$\mathrm{r} \leq 0$ & $\mathrm{r}=1$ & $45,544^{*}$ & $72,279^{*}$ \\
\hline
\end{tabular}

Fonte: Dados da pesquisa

* Significativo a $1 \%$ de significância [valores críticos em Osterwald-Lenum (1992)]. Modelo com constante restrita, ajustado com três defasagens.

Na Tabela 3, apresenta-se o coeficiente estimado para a matriz de relações contemporâneas do modelo VEC, especificada considerando que há relação contemporânea apenas do preço de exportação sobre o preço de mercado interno, e que os impactos dessas e das demais variáveis sobrea quantidade exportada ocorrem apenas em períodos posteriores. 0 sinal do coeficiente está de acordo com o esperado e é significativo.

Tabela 3 - Estimativa da matriz de relações contemporâneas

\begin{tabular}{|c|c|c|c|}
\hline \multicolumn{2}{|c|}{ Influência: } & \multirow{2}{*}{$\begin{array}{c}\text { Coeficiente } \\
\text { Estimado }\end{array}$} & \multirow{2}{*}{$\begin{array}{l}\text { Desvio } \\
\text { padrão }\end{array}$} \\
\hline Do & Sobre o & & \\
\hline pe & $p d$ & 0,4273 & 0,1521 \\
\hline
\end{tabular}

FONTE: Dados da pesquisa

As Figuras 3 a 6 mostram os efeitos de choques nas variáveis que compõem o modelo nos primeiros 12 períodos seguintes ao instante do choque inicial. Os resultados indicam que o efeito de uma variação do câmbio sobre o quantum exportado passa a ser expressivo no terceiro mês após o choque, tendo seu maior valor no quarto período (Figura 3). Uma variação de $1 \%$ na taxa de câmbio provoca, após quatro meses, uma variação de aproximadamente $2,18 \%$ nas exportações de açúcar. 0 preço externo tem um efeito positivo da ordem de $0,49 \%$ sobre 0 quanto exportado no segundo período, e um negativo no terceiro período (Figura 4). Essa variável, entre as consideradas no modelo, foi a que apresentou o menor efeito sobre as exportações.

No caso do preço interno e da renda, os maiores efeitos ocorrem no segundo e terceiro mês após o choque, respectivamente. Para o preço in- 
terno, a elasticidade para esse mês é de aproximadamente -1,36\% , e para renda, de -3,67 \% no terceiro período após o choque (Figuras 5 e 6).

Os resultados encontrados para o período sob análise mostraram que as variáveis relacionadas ao mercado doméstico têm grande influência sobre o quantum exportado de açúcar, para uma mesma variação percentual. Esse resultado sugere que as exportações de açúcar são grandemente afetadas pela conjuntura doméstica.

Figura 3 - Função de resposta do quantum exportado de açúcar a impulso na taxa de câmbio.

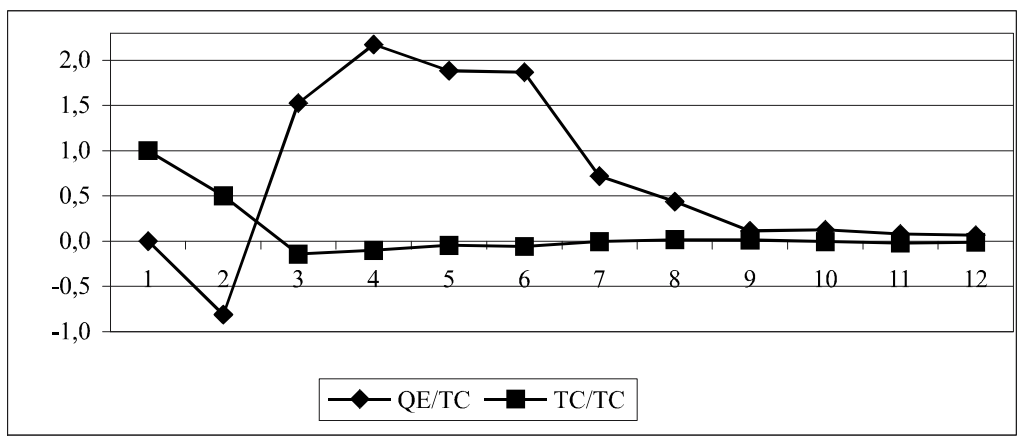

FONTE: Dados da pesquisa.

Figura 4 - Função de resposta do quantum exportado de açúcar a impulso no preço de exportação do produto.

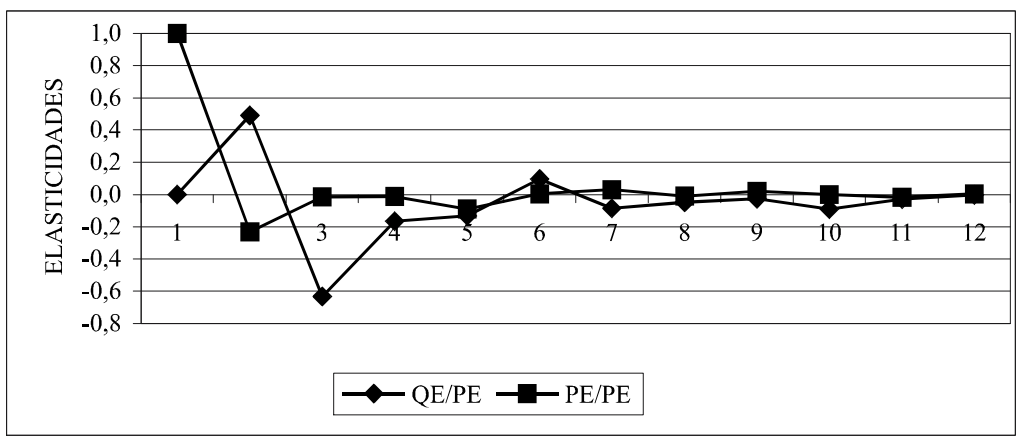

FONTE: Dados da pesquisa. 
Figura 5 - Função de resposta do quantum exportado de açúcar a impulso no preço doméstico do produto.

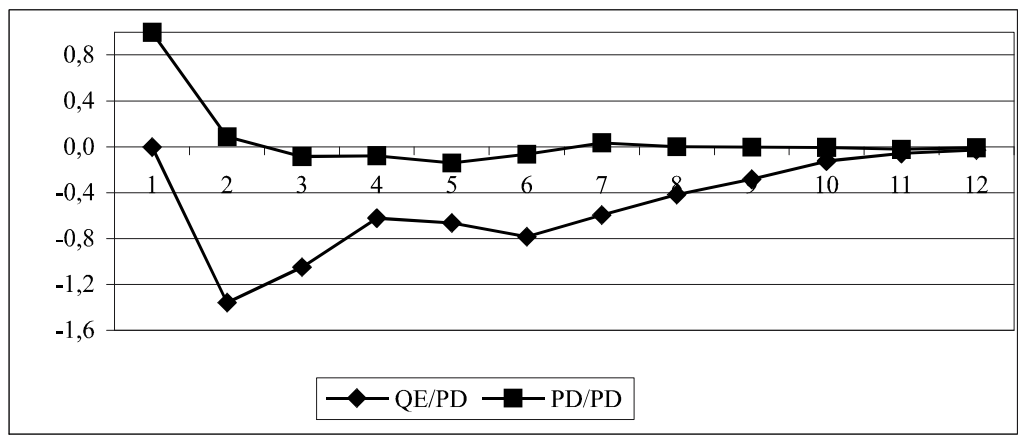

FONTE: Dados da pesquisa.

Figura 6 - Função de resposta do quantum exportado de açúcar a impulso na renda.

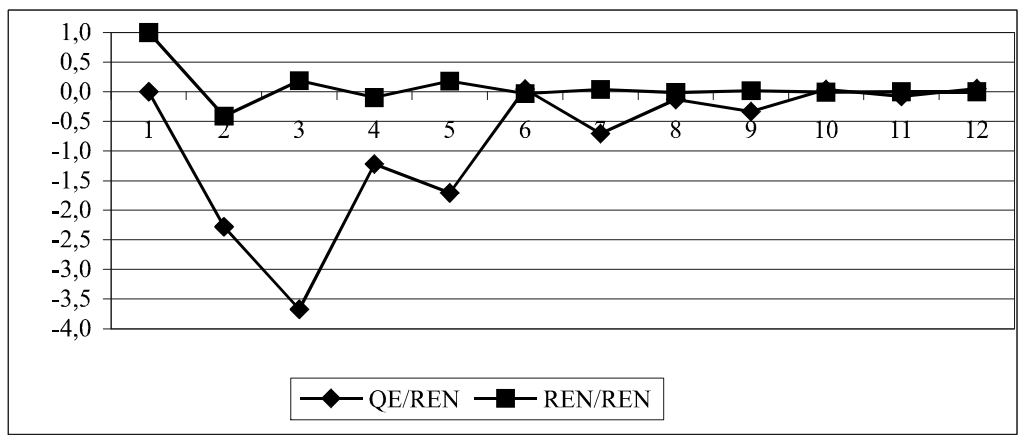

FONTE: Dados da pesquisa

Como mencionado anteriormente, a metodologia VAR permite avaliar o poder explanatório de cada variável do modelo sobre as demais através da decomposição da variância do erro de previsão. Na Tabela 4 tem-se a referida decomposição para a variável quantidade exportada de açúcar, as estimativas dos desvios-padrão dos erros, bem como a proporção desses erros atribuída a cada variável do modelo. Observa-se que o preço doméstico explica de $5,7 \%$ a $10,2 \%$ da variância do erro de previsão do quantum exportado e a renda de 3,5\% a 10,5\%, dependen- 
do do período considerado. O preço das exportações e a taxa de câmbio têm menor poder explicativo sobre o erro de previsão do quantum exportado, comparativamente às variáveis de mercado interno.

Tabela 4 - Decomposição histórica do erro de previsão do quantum exportado de açúcar

\begin{tabular}{ccccccc}
\hline Meses & Desvio padrão & $\boldsymbol{q} \boldsymbol{e}$ & $\boldsymbol{p} \boldsymbol{e}$ & $\boldsymbol{p d}$ & $\boldsymbol{t c}$ & $\boldsymbol{r e n}$ \\
\hline 1 & 0,387 & 100,000 & 0,000 & 0,000 & 0,000 & 0,000 \\
2 & 0,444 & 89,962 & 0,391 & 5,737 & 0,382 & 3,528 \\
3 & 0,505 & 81,028 & 0,798 & 7,059 & 1,341 & 9,774 \\
4 & 0,522 & 78,574 & 0,781 & 7,492 & 3,251 & 9,902 \\
5 & 0,535 & 75,837 & 0,763 & 8,084 & 4,519 & 10,796 \\
6 & 0,543 & 73,966 & 0,750 & 9,099 & 5,728 & 10,457 \\
7 & 0,547 & 73,137 & 0,748 & 9,708 & 5,855 & 10,551 \\
8 & 0,548 & 72,823 & 0,747 & 10,015 & 5,902 & 10,512 \\
9 & 0,548 & 72,665 & 0,746 & 10,156 & 5,894 & 10,539 \\
10 & 0,549 & 72,631 & 0,754 & 10,182 & 5,898 & 10,535 \\
11 & 0,549 & 72,624 & 0,755 & 10,187 & 5,899 & 10,535 \\
12 & 0,549 & 72,621 & 0,755 & 10,188 & 5,900 & 10,536 \\
\hline
\end{tabular}

FONTE: Dados da pesquisa.

Nas Tabelas 5 e 6 são apresentadas as decomposições da variância do erro de previsão do preço das exportações e do preço doméstico, respectivamente. Verifica-se que o preço doméstico explica aproximadamente $12 \%$ do erro de previsão do preço das exportações após o quarto mês, enquanto o preço das exportações explica aproximadamente $15 \%$ do erro de previsão do preço do mercado doméstico após esse período. Esse resultado relativamente expressivo entre o preço interno e de exportação é justificado pela interação existente nos anos recentes entre os mercados doméstico e internacional de açúcar. Vale lembrar que a relação contemporânea entre esses preços foi estimada em aproximadamente 0,43 , indicando uma relação expressiva entre eles. 
Tabela 5 - Decomposição histórica do erro de previsão do preço externo

\begin{tabular}{ccccccc}
\hline Meses & Desvio padrão & $\boldsymbol{q} \boldsymbol{e}$ & $\boldsymbol{p} \boldsymbol{p}$ & $\boldsymbol{p d}$ & $\boldsymbol{t c}$ & $\boldsymbol{r e n}$ \\
\hline 1 & 0,056 & 0,000 & 100,000 & 0,000 & 0,000 & 0,000 \\
2 & 0,063 & 0,240 & 85,838 & 8,701 & 5,053 & 0,167 \\
3 & 0,063 & 0,791 & 83,466 & 9,110 & 5,600 & 1,034 \\
4 & 0,065 & 0,843 & 80,478 & 11,933 & 5,518 & 1,228 \\
5 & 0,065 & 0,844 & 79,044 & 11,842 & 5,858 & 2,413 \\
6 & 0,065 & 0,843 & 78,918 & 11,953 & 5,854 & 2,432 \\
7 & 0,066 & 0,856 & 78,840 & 11,956 & 5,921 & 2,428 \\
8 & 0,065 & 0,869 & 78,496 & 12,244 & 5,948 & 2,444 \\
9 & 0,066 & 0,870 & 78,493 & 12,239 & 5,954 & 2,444 \\
10 & 0,066 & 0,877 & 78,457 & 12,266 & 5,956 & 2,444 \\
11 & 0,066 & 0,877 & 78,455 & 12,265 & 5,959 & 2,445 \\
12 & 0,066 & 0,877 & 78,449 & 12,267 & 5,958 & 2,449 \\
\hline
\end{tabular}

FONTE: Dados da pesquisa.

Tabela 6 - Decomposição histórica do erro de previsão do preço doméstico

\begin{tabular}{ccccccc}
\hline Meses & Desvio padrão & $\boldsymbol{q} \boldsymbol{e}$ & $\boldsymbol{p e}$ & $\boldsymbol{p d}$ & $\boldsymbol{t} \boldsymbol{c}$ & $\boldsymbol{r e n}$ \\
\hline 1 & 0,082 & 0,000 & 8,682 & 91,318 & 0,000 & 0,000 \\
2 & 0,086 & 2,051 & 13,959 & 80,442 & 3,542 & 0,006 \\
3 & 0,089 & 3,513 & 13,712 & 78,515 & 4,041 & 0,218 \\
4 & 0,090 & 3,908 & 14,598 & 76,936 & 4,319 & 0,239 \\
5 & 0,091 & 3,852 & 14,326 & 76,983 & 4,326 & 0,513 \\
6 & 0,091 & 3,973 & 14,356 & 76,851 & 4,306 & 0,515 \\
7 & 0,091 & 3,967 & 14,363 & 76,805 & 4,324 & 0,540 \\
8 & 0,091 & 3,959 & 14,382 & 76,641 & 4,455 & 0,562 \\
9 & 0,091 & 3,959 & 14,395 & 76,608 & 4,474 & 0,564 \\
10 & 0,091 & 3,959 & 14,396 & 76,606 & 4,474 & 0,565 \\
11 & 0,091 & 3,958 & 14,392 & 76,612 & 4,472 & 0,566 \\
12 & 0,091 & 3,957 & 14,393 & 76,609 & $4, .474$ & 0,566 \\
\hline
\end{tabular}

FONTE: Dados da pesquisa.

Estes resultados, em termos gerais, corroboram os encontrados por Barros, et al. (2002). No entanto, diferentemente daquele trabalho, a metodologia aqui empregada permite que se observe, além da magnitude do efeito do choque, também a duração deles. Esta informação é importante para fundamentar a elaboração de análises prospectivas. 


\section{Considerações Finais}

O modelo teórico utilizado para a definição das condicionantes das exportações de açúcar, no qual se considera que a oferta de exportação brasileira depende da diferença entre a oferta e demanda doméstica, apresenta-se adequado para a análise. 0 preço e a renda doméstica, variáveis que refletem diretamente as condições de mercado interno, foram de grande importância na determinação das exportações brasileiras de açúcar, sendo o efeito defasado (subseqüentes ao choque) dessas variáveis sobre o quantum exportado bastante expressivo. Do mesmo modo, há estímulo às exportações de açúcar quando a moeda nacional sofre desvalorização em relação à moeda americana. Embora o efeito da variação da taxa de câmbio sobre o quantum exportado não seja grande no primeiro e no segundo períodos após um choque, ele passa a ser bastante expressivo após três meses.

0 preço das exportações, que reflete as condições de oferta e demanda prevalecentes no mercado internacional, embora tenha apresentado menor elasticidade comparativamente às variáveis de mercado interno, teve algum grau de influência sobre o quantum exportado de açúcar, sabendo-se que ele é um sinalizador do potencial de absorção do produto naquele mercado. Segundo Barros et al. (2002), maiores preços internacionais indicam excesso de demanda nesse mercado, 0 que possibilita a expansão das exportações brasileiras.

Acredita-se que políticas setoriais que levem a ganhos em produção e produtividade, por terem caráter mais independente do que as políticas macroeconômicas que afetam de forma diferenciada vários segmentos da economia, sendo de difícil implementação, parecem ser instrumentos eficazes para possibilitar a geração de excedentes exportáveis. A situação econômica favorável das unidades produtoras de açúcar, que se refletem nos tratos culturais da lavoura da cana-de-açúcar e na produção de açúcar, podem contribuir para a dinamização do setor exportador açucareiro brasileiro.

Deve-se considerar, também, que a flexibilidade existente na agroindústria canavieira quanto à possibilidade de se produzir açúcar ou álcool, dependendo das condições de mercados desses produtos, deve influir sobremaneira na definição dos excedentes exportáveis de açú- 
car. Nesse sentido, seria importante a introdução do preço do álcool no modelo ajustado para a análise das exportações de açúcar, o que não foi feito em função da indisponibilidade desses dados para a maior parte do período de abrangência do estudo. É importante lembrar que o preço do álcool foi por muito tempo tabelado e, portanto, a série dessa variável apresenta pouca variabilidade, justificando a sua não inclusão na função de oferta de açúcar.

\section{Referências Bibliográficas}

AGUIAR, D. Custo, risco e margem de comercialização de arroz e feijão no Estado de São Paulo: análise dinâmica e testes de modelos alternativos. Piracicaba, 1994. Tese (Doutorado) - Escola Superior de Agricultura "Luiz de Queiroz"/ Universidade de São Paulo.

ALVES, L.R.A. Transmissão de preços entre produtos do setor sucroalcooleiro do Estado de São Paulo. Piracicaba, 2002. Dissertação (M estrado) - Escola Superior de Agricultura "Luiz de Queiroz"/ Universidade de São Paulo.

ASSOCIAÇÃO DE PRODUTORES DE ÁLCOOL E AÇÚCAR DO ESTADO DO PARANÁ - ALCOPAR. Mercado de açúcar. http:// www.alcopar.org.br/ estatdiv/ macucar.htm (15 abr. 2003)

BACCHI, M.R.P.; BURNQUIST, H.L. Transmissão de preços entre os segmentos produtivos da pecuária de corte brasileira. In: CONGRESSO BRASILEIRO DE ECONOMIA E SOCIOLOGIA RURAL, Foz de Iguaçu, PR, 1999. Anais, Brasília : SOBER, 1999.

BARROS, G.S. de C. Formação de preços no setor de frango de corte no Brasil. Relatório de Pesquisa. Escola Superior de Agricultura Luiz de Queiroz/USP. 1994.

BARROS, G.S. de C.; BACCHI, M.R.P.; BURNQUIST, H.L. Estimação de equações de oferta de exportação de produtos agropecuários para o Brasil (1992/2000). Texto para Discussão, Brasília: IPEA, n.865, mar. 2002.

BARROS, G.S. de C.; BITTENCOURT, M.V.L. Formação de preços sob oligopsônio: o mercado de frango em São Paulo. Revista Brasileira de Economia, Rio de Janeiro, v.51, n.2, p.181-199, 1997.

BARROS, W.J de. Análise econométrica dos mercados interno e de exportação de açúcar. Viçosa, 1975. Dissertação (Mestrado) - Universidade Federal de Viçosa. 
BERNANKE, B.S. Alternative explanations of the money-income correlation. Carnegie-Rochester Conference Series on Public Policy, v.25, p.49-100, 1986.

BURNQUIST, H.L.(Coord.); BACCHI, M.R.P.; FIGUEIREDO, N.M.S. de; VIEIRA, W. da C. Liberalização comercial: um fator de desenvolvimento do setor agrícola brasileiro. Série Estudos de Política Agrícola, Relatório de Pesquisa, Brasília: IPEA, n.14, 1994.

BURNQUIST, H.L.; MIRANDA, S.H.G. de Desempenho recente das exportações brasileiras de açúcar: uma abordagem de "market-share" constante. Revista de Economia e Sociologia Rural, Brasília, v.37, n.3, p. 69-90, jul.-set. 1999.

CARVALHO, A.; NEGRI, J .A. Estimação de equações de importação e exportação de produtos agropecuários para o Brasil (1977/1998). Texto para Discussão, Brasília: IPEA, n.698, 2000.

CARVALHO, F.C.; BRANDT, S.A. Avaliação da política de estabilização de preços no mercado de exportação de açúcar do Brasil. Revista de Economia Rural, Brasília, v.25, n.3, p.357-365, sul.-set. 1987.

CASTRO, A.S; CAVALCANTI, M.A. Estimação de equações de exportação e importação para o Brasil - 1955/95. Texto para Discussão, Brasília: IPEA, n.469, mar. 1997.

CAVALCANTI, M.A.; RIBEIRO, M.A.F.H. As exportações no período 1977/96: desempenho e determinantes. Texto para Discussão, Brasília: IPEA, n.545, fev. 1998.

DICKEY, D.A.; FULLER, W.A. Distribution of the estimator for auto-regressive time series with a unit root. J ornal of the American Statistical Association, v.74, n.366, p.427-431, Jun. 1979.

DICKEY, D.A.; FULLER, W.A. Likelihood ratio statistics for autoregressivetime series with a unit root. Econometrica, Chicago, v.49, n.4, p.1057-1072, J ul. 1981.

HAMILTON, J.D. Time series analysis. Princenton University Press. Princenton: New Yersey. 1994.

HARVEY, A. The econometric analysis of time series. MIT Press. Cambridge, Massachusetts. 1990.

HOFFMANN, R. Elasticidades-renda das despesas e do consumo físico de ali- 
mentos no Brasil metropolitano em 1995/96. Agricultura em São Paulo, São Paulo, v.47, n.1, p.111-122, 2000.

J OHANSEN, S. Statistical analysis of cointegration vectors. J ournal of Economic Dynamics and Control, v.12, p.231-254, 1988.

J OHANSEN, S.; JUSELIUS, K. Maximum likelihood estimation and inference on cointegration - with aplications to the demand for money. Oxford Bulletin of Economics and Statistics, v.52, p.169-219, 1990.

LÜTKEPOHL, H. Introduction to multiple time series analysis. Springer-Verlag. Berlin. 1991.

MARJ OTTA-MAISTRO, M.C. Ajustes nos mercados de álcool e gasolina no processo de desregulamentação. Piracicaba, 2002. 180p. Tese (Doutorado) - Escola Superior de Agricultura "Luiz de Queiroz", Universidade de São Paulo.

MINISTÉRIO DO DESENVOLVIMENTO, INDÚSTRIA E COMÉRCIO/SECRETARIA DE COMÉRCIO EXTERIOR - MDIC/SECEX. http:// www.mdic.gov.br (15 abr. 2003)

MIRANDA, S.H.G. de. Quantificação dos efeitos das barreiras não-tarifárias sobre as exportações brasileiras de carne bovina. Piracicaba, 2001. Tese (Doutorado) - Escola Superior de Agricultura "Luiz de Queiroz", Universidade de São Paulo.

MORAES, M.A.F.D. de. A desregulamentação do setor sucroalcooleiro do Brasil. Americana: Caminho Editorial, 2000. 238p.

MYERS, R.J .; PIGGOTT, R.R.; TOMEK, W.G. Esthimating sources of fluctuations in the australian wool market: an application of VAR methods. Australian J ournal of Agricultural Economics, v.34, p.242-262, 1990.

ONUNKWO, I.M.; EPPERSON, J.E. Export demand for U.S. Pecans: impacts of U.S. export promotion programs. In: ANNUAL MEETING OF THE AMERICAN AGRICULTURAL ECONOMICSASSOCIATION IN NASHVILLE. Tennessee. Aug. 1999, p.8-11.

OSTERWALD-LENUM, M. A note with quantiles of the asymptotic distribution of the maximum likelihood cointegration rank test statistics. Oxford Bulletin of Economics and Statistics, v.53, n.3, p.461-472, 1992.

REIS, J.N.P.; CRESPO, J.E.Q. Um modelo econométrico para as exportações de açúcar do Brasil. Agricultura Em São Paulo, São Paulo, v.45, n.1, p.17-32, 1998. 
SENHADJI, A.S.; MONTENEGRO, C.E. Time series analysis of export demand equations: a cross-country analysis. IMF Staff Papers, v.46, n.3, p.259-273, Sep./Dec. 1999.

STALDER, S.H.G.; BURNQUIST, H.L. Exportações de açúcar no Brasil: uma abordagem de elasticidade. In: CONGRESSO BRASILEIRO DE ECONOMIA E SOCIOLOGIA RURAL, 35., NATAL, 1997. Anais. Brasília: SOBER, 1997. p.289.

UNIÃO DA AGROINDÚSTRIA CANAVIEIRA DO ESTADO DE SÃO PAULO - UNICA. Sociedade. http:// www.unica.com.br/ pages/ sociedade_mercado1.asp (20 out. 2002)

UNITED STATES DEPARTMENT OF AGRICULTURE - USDA. Sugar: world markets and trade. http:// www.fas.usda.gov/ htp/ sugar/ sugar.html (15 Nov. 2002)

VEIGA FILHO, A. O dilema da "escolha de sofia" nas exportações de açúcar pelo Brasil. Informações Econômicas, São Paulo, v.30, n.9, p.53-59, set. 2000.

ZINI J R., A.A. Funções de exportação e de importação para o Brasil. Pesquisa e Planejamento Econômico, Rio de J aneiro, v.18, p.615-662, 1988.

Recebido em setembro de 2003 e revisto em fevereiro de 2004. 